\title{
Prediction of novel miRNAs and associated target genes in Glycine max
}

\author{
Trupti Joshi ${ }^{1}$, Zhe Yan ${ }^{2}$, Marc Libault ${ }^{2}$, Dong-Hoon Jeong ${ }^{3}$, Sunhee Park ${ }^{3}$, \\ Pamela J Green ${ }^{3}$, D Janine Sherrier ${ }^{3}$, Andrew Farmer ${ }^{4}$, Greg May ${ }^{4}$, \\ Blake C Meyers ${ }^{3}$, Dong $\mathrm{Xu}^{1}$ and Gary Stacey*2
}

\begin{abstract}
Addresses: ${ }^{1}$ Digital Biology Laboratory, Computer Science Department and Christopher S. Bond Life Sciences Center, University of Missouri, Columbia, MO 65211, USA, ${ }^{2}$ Division of Plant Sciences, National Center for Soybean Biotechnology, C.S. Bond Life Sciences Center, University of Missouri, Columbia, MO 65211, USA, ${ }^{3}$ Department of Plant and Soil Sciences and Delaware Biotechnology Institute, University of Delaware, Newark, DE 19711, USA and ${ }^{4}$ National Center for Genome Resources, 2935 Rodeo Park Drive East, Santa Fe, NM 87505, USA

E-mail: Trupti Joshi - joshitr@missouri.edu; Zhe Yan - yanzh@missouri.edu; Marc Libault - libaultm@missouri.edu; Dong-Hoon Jeong - jeong@dbi.udel.edu; Sunhee Park - spark@dbi.udel.edu; Pamela J Green - green@dbi.udel.edu;

D Janine Sherrier - sherrier@dbi.udel.edu; Andrew Farmer - adf@ncgr.org; Greg May - gdm@ncgr.org; Blake C Meyers - meyers@dbi.udel.edu; Dong Xu -xudong@missouri.edu; Gary Stacey*-staceyg@missouri.edu

*Corresponding author
\end{abstract}

from The Eighth Asia Pacific Bioinformatics Conference (APBC 2010)

Bangalore, India I8-2I January 2010

Published: 18 January 2010

BMC Bioinformatics 2010, II (SuppI I):SI4 doi: 10.II86/I47I-2I05-II-SI-SI4

This article is available from: http://www.biomedcentral.com//47I-2I05/II/SI/SI4

(C) 2010 Joshi et al; licensee BioMed Central Ltd.

This is an open access article distributed under the terms of the Creative Commons Attribution License (http://creativecommons.org/licenses/by/2.0), which permits unrestricted use, distribution, and reproduction in any medium, provided the original work is properly cited.

\begin{abstract}
Background: Small non-coding RNAs (2I to 24 nucleotides) regulate a number of developmental processes in plants and animals by silencing genes using multiple mechanisms. Among these, the most conserved classes are microRNAs (miRNAs) and small interfering RNAs (siRNAs), both of which are produced by RNase III-like enzymes called Dicers. Many plant miRNAs play critical roles in nutrient homeostasis, developmental processes, abiotic stress and pathogen responses. Currently, only 70 miRNA have been identified in soybean.

Methods: We utilized Illumina's SBS sequencing technology to generate high-quality small RNA (sRNA) data from four soybean (Glycine max) tissues, including root, seed, flower, and nodules, to expand the collection of currently known soybean miRNAs. We developed a bioinformatics pipeline using in-house scripts and publicly available structure prediction tools to differentiate the authentic mature miRNA sequences from other sRNAs and short RNA fragments represented in the public sequencing data.

Results: The combined sequencing and bioinformatics analyses identified 129 miRNAs based on hairpin secondary structure features in the predicted precursors. Out of these, 42 miRNAs matched known miRNAs in soybean or other species, while 87 novel miRNAs were identified. We also predicted the putative target genes of all identified miRNAs with computational methods and verified the predicted cleavage sites in vivo for a subset of these targets using the 5' RACE method.
\end{abstract}


Finally, we also studied the relationship between the abundance of miRNA and that of the respective target genes by comparison to Solexa cDNA sequencing data.

Conclusion: Our study significantly increased the number of miRNAs known to be expressed in soybean. The bioinformatics analysis provided insight on regulation patterns between the miRNAs and their predicted target genes expression. We also deposited the data in a soybean genome browser based on the UCSC Genome Browser architecture. Using the browser, we annotated the soybean data with miRNA sequences from four tissues and cDNA sequencing data. Overlaying these two datasets in the browser allows researchers to analyze the miRNA expression levels relative to that of the associated target genes. The browser can be accessed at http://digbio. missouri.edu/soybean mirna/.

\section{Background}

Many classes of 18-30 nt small non-coding RNAs (sRNAs) can be characterized based on their functions in gene regulation and epigenetic control in plants, animals and fungi $[1,2]$.

Identification of the complete set of miRNAs and other small regulatory RNAs in organisms is essential with regard to our understanding of genome organization, genome biology, and evolution [3]. There are three important classes of endogenous small RNAs in plants, animal or fungi: micro RNAs (miRNAs), short interfering RNAs (siRNAs) and piwi-interacting RNAs (piRNAs). In plants, there are no known piRNA.

MicroRNAs (miRNAs) are small 18-24 nucleotide regulatory RNAs that play very important roles in posttranscriptional gene regulation by directing degradation of mRNAs or facilitating repression of targeted gene translation $[4,5]$. While siRNA are processed from longer double stranded RNA molecules and represent both strands of the RNA, miRNAs originate from hairpin precursors formed from one RNA strand [6,7]. The hairpin precursors (pre-miRNA) are typically around $\sim 60-70$ bp in animals, but somewhat larger, 90-140 bp in plants. In plants, helped by RNA polymerase II, miRNA gene is first transcribed into pri-miRNA. The pri-miRNAs are cleaved to miRNA precursors (pre-miRNA), which form a characteristic hairpin structure, catalyzed by Dicerlike enzyme (DCL1) [7,8]. The pre-miRNA is further cleaved to a miRNA duplex (miRNA:miRNA*), a short double-stranded RNA (dsRNA) [9]. The dsRNA is then exported to cytoplasm by exportin-5. Helped by AGO1, single-strand mature miRNA will form a RNA-protein complex, named RNA-induced silencing complex (RISC), which negatively regulates gene expression by inhibiting gene translation or degrading mRNAs by perfect or nearperfect complement to target mRNAs $[10,11]$.

Although some soybean miRNA were previously identified [12], the number was small and, therefore, the identification of all soybean miRNAs is far from complete. The aim of this study is to expand the collection of miRNAs expressed in soybean by using a deep sequencing approach with the Illumina Solexa platform. Towards this, we generated Solexa cDNA sequencing data for root, nodule and flower tissues since they are all relevant soybean organs to various studies in legume biology and due to their impact on soybean yield. One of the legume-specific traits is the symbiosis existing between the legume root and soil bacteria leading to the nodule. We think the small RNA content of soybean nodules needs to be established since research in other legume species showed a role for small RNA in nodule development $[13,14]$. Root tissue is another important organ to analyze due to its role in nutrient-water absorption, which is clearly important to soybean yield. Finally, we selected flower for its direct impact on soybean seed yield. We constructed the small RNA libraries, prepared from these four different soybean tissues and each library was sequenced individually, generating a total of over one million sequences per library. We developed a bioinformatics pipeline using in-house developed scripts and other publicly available RNA structure prediction tools to differentiate the authentic mature miRNA sequences from other small RNAs and short RNA fragments represented in the sequencing data. We also conducted a detailed analysis of predicted miRNA target genes and correlated the miRNA expression data to that of the corresponding target genes using Solexa cDNA sequencing data.

\section{Methods}

\section{Sources of sequences and assemblies}

Illumina's SBS sequencing technology was utilized to generate high-quality reads from four different soybean tissues, including root, seed, flower, and nodule. The Gmax1.01 release version genomic sequences and gene model predictions of Williams 82 soybean genome were acquired from Phytozome [15] and used as a reference genome. 


\section{Small RNA library construction and SBS sequencing} Soybean (Glycine max L. Merr.) cultivar Williams 82 were planted in $5 \mathrm{~L}$ pots containing a mixture of two parts Metro-Mix 360 (Scotts-Sierra Horticultural Products Co., Marysville, Ohio) and 1 part vermiculite (Strong-Lite Medium Vermiculite, Sun Gro Horticulture Co, Seneca Illinois). Plants were grown under controlled environmental conditions in a greenhouse, with a temperature regime of $22 \pm 3{ }^{\circ} \mathrm{C} /$ day and $20 \pm 3{ }^{\circ} \mathrm{C} /$ night, and relative humidity ranging from $45 \%$ to $65 \%$ throughout the day/night cycle. Sunlight was supplemented with metal halide lamps, set to a 15-h day, 9-h night cycle (lights on at $700 \mathrm{~h}$ ). The soil mixture was kept moist by an automated drip irrigation system, which delivered nutrient solution twice a day (younger plants) or three times a day (older plants). The nutrient solution contained the following concentrations of mineral salts: $1 \mathrm{mM} \mathrm{KNO}_{3}, 0.4 \mathrm{mM} \mathrm{Ca}\left(\mathrm{NO}_{3}\right)^{2}, 0.1$ $\mathrm{mM} \mathrm{MgSO}_{4}, 0.15 \mathrm{mM} \mathrm{KH}_{2} \mathrm{PO}_{4}$ and $25 \mu \mathrm{M} \mathrm{CaCl}_{2}, 25 \mu \mathrm{M}$ $\mathrm{H}_{3} \mathrm{BO}_{3}, 2 \mu \mathrm{m} \mathrm{MnSO}, 2 \mu \mathrm{M} \mathrm{ZnSO}{ }_{4}, 0.5 \mu \mathrm{M} \mathrm{CuSO}_{4}, 0.5$ $\mu \mathrm{M} \mathrm{H} 2 \mathrm{MoO}_{4}, 0.1 \mu \mathrm{M} \mathrm{NiSO}{ }_{4}, 1 \mu \mathrm{M} \mathrm{Fe}(\mathrm{III})-/ \mathrm{N} /, / \mathrm{N}^{\prime}-/$ ethylenebis [2-(2-hydroxyphenyl)-glycine]. Flowers were collected at -2 to 2 days after anthesis. Seeds were harvest at 20 days post anthesis. Root tissues were collected from 3 weeks-old soybean seedlings, which were grown with nitrogen and $1 / 2$ lullien [16] solution in cassions. To collect nodules, soybean plants were grown in aeropontic growth champers for 7 days in $1 / 2 \times$ Lullien medium [16], with no nitrogen and inoculated with Bradyrhizobium japonicum USDA110 to induce nodule formation. Nodules were collected 7, 14, and 21 days after inoculation and pooled for RNA isolation. Total RNA of different tissues were isolated by TRIzol reagents (Invitrogen). Small RNA libraries were constructed and sequenced as previously described [17].

\section{Bioinformatics analyses of sequencing data}

The sequencing reads were generated for all four libraries along with base quality score information. The sequences were initially trimmed for adapter sequences with an in-house trimming procedure and raw abundance read counts were calculated for all unique sequences for each library individually. This final set of reads and corresponding read counts for each library were all combined to generate a list of unique sequences, which are referred to as sequence tags henceforth. These sequence tags were then mapped to the soybean genome assembly using the stand-alone version of Megablast software [18], and the corresponding matching chromosome number, positions and strand were recorded for those mapped to the genome with no mis-matches in sequence tags. The sequence tags not passing this mapping criterion were excluded from further analysis.

We further analysed these 1.2 million, mapped sequence tags against the gene model predictions and identified and updated the information for any sequence tags which overlapped with the coding gene positions on the genome. We also predicted the tRNAs in the soybean genome using tRNAscan-SE version 1.21 software [19] and also looked for sequence similarities using BLAST for the tRNAs in other genomes in the Genomic tRNA database [20]. These searches identified 1200 tRNA predicted in soybean. The 2469 sequence tags that mapped to the known tRNAs positions were filtered from further analysis. We also performed rRNA predictions in soybean using RNAmmer version 1.2 [21]. Subsequently, the sequence tags that mapped to the 620 known rRNAs in soybean were also removed before further analysis. Table 1 shows the number of sequence tags removed due to overlap with the tRNA and rRNA positions per library.

The raw abundance values for sequence tags in every library were normalized into corresponding transcripts per million (TPM) abundance values using

$$
\text { TPM abundance }=(\text { raw value } / \# \text { sum_use })^{*} n \_ \text {base }
$$

where n_base is a million $(1,000,000)$; \#sum_use is number of tags found to hit genomic positions of non-tRNA and non-rRNA regions. To ensure that we were working with good quality and expressed small RNA sequences, we further removed any sequence tags where the sum of TPM abundance from all the four libraries was <20 TPM [22]. At

Table I: Statistics of sequenced tags matching genome, tRNA and rRNA

\begin{tabular}{lllll}
\hline & Root & Nodule & Flower & Seed \\
\hline Tags with genome hits & 674226 & 665947 & 686029 & 853162 \\
Distinct tags with genome hits & 172795 & 287855 & 288804 & 350869 \\
Tags with genome hits unique to library & 102947 & 198520 & 185554 & 244069 \\
Tags with tRNA hits & 78680 & 40622 & 41114 & 106561 \\
Distinct tags with tRNA hits & 756 & 423 & 611 & 719 \\
Tags with rRNA hits & 2367 & 2714 & 1754 & 1618 \\
Distinct tags with rRNA hits & 524 & 644 & 479 & 437 \\
\# Sum_use (tags with non tRNA, rRNA genomic hits) & 595546 & 625325 & 644915 & 746601 \\
\hline
\end{tabular}


the conclusion of these filtering steps, 113,399 sequence tags were considered in our further analysis.

\section{miRNA identification}

We extracted $200 \mathrm{bp}$ upstream and downstream genomic sequences for all the sequence tags that passed the $>20$ TPM abundance filtering criterion and further predicted the hairpin-like RNA secondary structures for all. The secondary structure was predicted by DINAMelt program using default RNA3.0 parameters [23]. To ensure the stem-loop precursor could be precisely processed into mature miRNA, the predicted structures were examined according to the following criteria [8]:

i. The candidate miRNA and miRNA* should come from opposite stem-arms and must be entirely within the arm of the hairpin;

ii. miRNA::miRNA* duplex mismatches were restricted to four or fewer;

iii. The frequency of asymmetric bulges is restricted to less than one and the size should be less than 2 bases.

Following these stringent filtering criteria, we identified 129 putative miRNA sequence tags that were compared against the downloaded miRBase [24], containing known miRNA sequences for soybean and other genomes, using FASTA to identify the already known miRNA and differentiate them from the novel miRNA. We identified a total of 129 putative miRNA sequences, of which 42 matched the already known miRNAs in miRBase while 87 were novel miRNAs.

\section{miRNA family assignment}

The 129 predicted miRNA sequences were further assigned to miRNA families using sequence similarity to other known miRNA (including already known soybean miRNA) in the miRBase database. The putative miRNA sequences, along with the known soybean miRNA sequences, were used for multiple alignments using ClustalW [25] and the miRNA families were assigned based on the dendogram tree. 42 miRNAs were assigned families based on matches to known miRNAs, while the 87 novel miRNAs will get new families using miRBase Registry.

\section{miRNA target gene prediction and experimental validation}

The 129 predicted miRNA sequences were later utilized for miRNA target identification using an in-house plant target prediction program following standard rules of miRNA-mRNA interactions $[26,27]$. The filtering criteria were: a mismatch is given a score of 1 , a wobble $(\mathrm{G}: \mathrm{U}$ mismatch) is given a score of 0.5 , and a bulge is given a score of 2 . The final entry score was set to 4 . At the same time, positions 10 and 11 of miRNA must perfectly match to its target, and there should be no more than one mismatch in miRNA position 2 to 9. Overall, we identified 603 target genes for 78 of the identified miRNA. Further analysis showed that target genes could be predicted for $~ 73 \%$ of known miRNAs and $~ 54 \%$ of novel miRNAs. No targets could be predicted for the remaining $\sim 26 \%$ of known miRNA and $\sim 45 \%$ of novel miRNA using the above stringent score cutoff. To validate the miRNA-cleavage site, PolyA RNA was extracted and a 5'-RACE reaction was performed using a Gene Racer core kit (Invitrogen, Carlsbad, CA) following previously published methods [12].

\section{Data display and data integration}

We deposited the data in a soybean genome browser based on the architecture provided by USCS genome browser. The Gmax1.01 release version genomic sequences and gene model predictions were downloaded from Phytozome and formatted to be used as annotations for our soybean genome browser. The mapped chromosome number and position information of the four small RNA library sequence tags was converted into a BED format and uploaded to the browser as user tracks. The final putative miRNA list was also converted to BED format and also color-coded based on expression pattern, as well as miRNA length.

\section{Solexa transcriptome data analysis and integration}

We also generated Solexa cDNA sequencing data for three out of the four soybean tissues (root, nodule and flower) that were used for small RNA library construction. In addition, we also have Solexa cDNA reads derived from mRNA isolated from green pods. The raw sequencing reads for the four tissues were aligned to the soybean reference genome acquired from Phytozome using the MAQ-0.6.6 version software [28]. The aligned read positions were further converted into the WIG format and uploaded to the soybean genome browser as user tracks for the four tissues individually. The transcriptomic data for the libraries were normalized against the total number of soybean reads identified in each tissue and analysed to generate the expression abundance values for all the genes in soybean. The expression values of the miRNA were compared against the miRNA target gene abundance values to provide some valuable insight into the predicted target gene regulation by the respective miRNA. We calculated Spearman correlation coefficients between the two and looked specifically into any miRNA-target gene pairs with a strong negative correlation.

\section{Results}

\section{Overview of small RNA sequencing results}

The sequencing reads for all four libraries were combined and a unique list of sequence tags was generated 


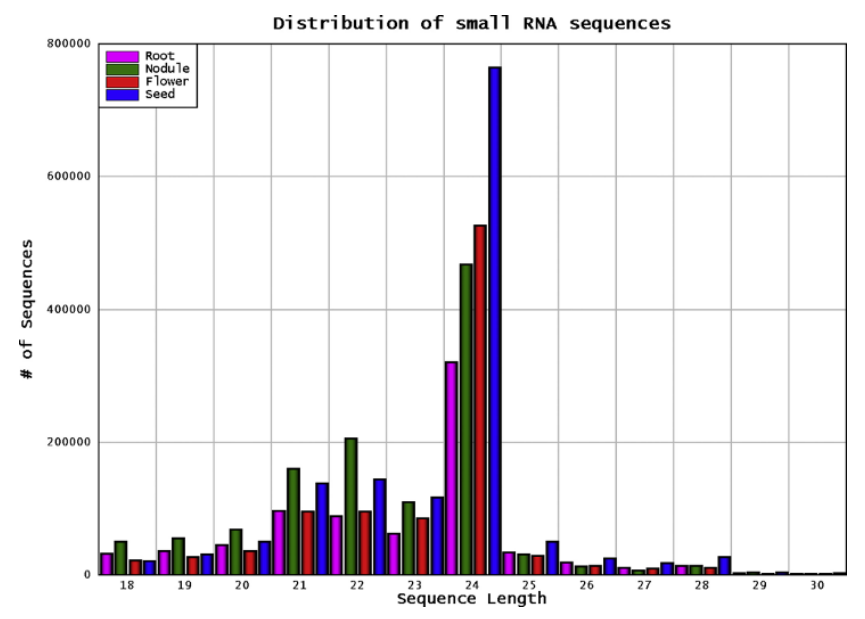

Figure I

Distribution of sequence reads in the small RNA libraries. Distribution of the various lengths of the small RNA sequences in the four libraries examined. The majority of the reads are 21, 22 and 24 nucleotides in length.

with the corresponding raw abundance read counts, for simplicity. Although the total combined sequencing data had over 1 million reads per library, the unique sequence tags were less than that. This highlights the fact that, while there are some common sequence tags being expressed in all or multiple libraries, there are some specifically present only in some libraries. The sequence tags varied in length from $18 \mathrm{bp}$ through $30 \mathrm{bp}$ as seen in Figure 1, with the highest abundance being around the lengths 21, 22 and 24 bp. Some of the variability in small RNA length may be the result of artefacts introduced by the cloning, trimming and/or sequencing techniques. The seed tissue library followed by the flower library had the largest number of sequencing reads and sequence tags with perfect genomic mapping, with 244,069 and 185,554 unique sequence tags in each library, respectively.

\section{Novel and known miRNA identification}

The bioinformatic analyses identified 129 miRNAs based on similarities to conserved known miRNAs, as well as novel miRNAs predicted from their hairpin secondary structure features derived from genomic sequences. The list of the 129 predicted miRNAs can be found in the Additional File 1. Figure 2 shows the hairpin structure of a predicted miRNA. Figure 3 shows the ranked, expression values for the four, different soybean tissues for each of the 129. Based on our filtering criteria, the novel miRNAs identified not only had to have a sum of expression $>20$ TPM from the 4 small RNA libraries but also could not overlap with the genomic loci of already

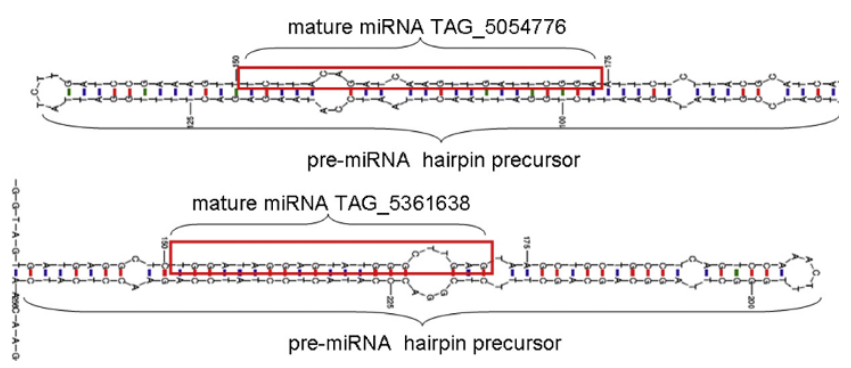

Figure 2

miRNA hairpin structure. The hairpin structure of the pre-miRNA precursor predicted using the Quickfold. The mature miRNA sequences TAG_5054776 and TAG_536I638 are highlighted in red, respectively.
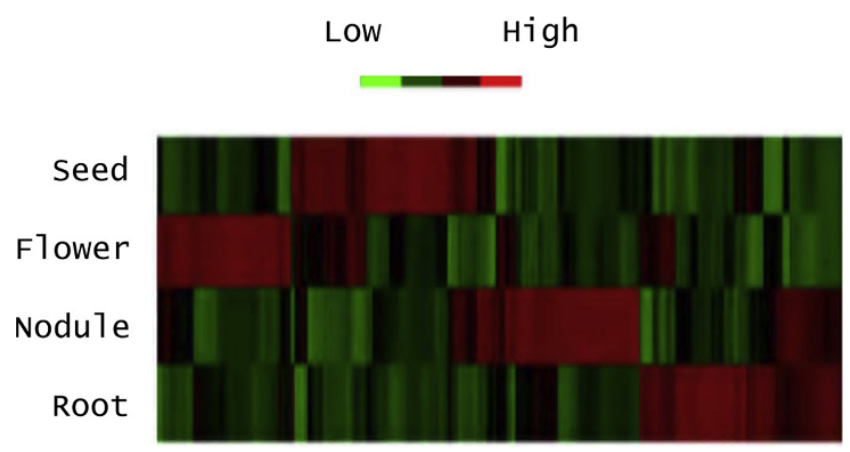

\section{Figure 3}

miRNA expression pattern. The expression pattern of the 129 predicted miRNA across the four small RNA libraries including root, nodule, flower and seed. The expression patterns were clustered together based on the expression pattern across libraries. The low and high expression levels are shown in green and red respectively.

annotated soybean miRNAs or other classes of noncoding RNAs. The resulting set of sequences and their respective RNA structures were analyzed to distinguish genuine miRNA precursors from other RNAs that contain similar RNA structures. We also observed that the sequenced tags were comprised of both the miRNA sequence as well as the miRNA* sequence, although the miRNA* was certainly less abundant in all four libraries than the miRNA. In total, the miRNA* sequences were identified for 105 predicted miRNA amongst all the sequenced libraries.

\section{miRNA target gene prediction}

We computationally predicted putative target genes for the 129 identified miRNAs. We identified overall 603 targets from 78 miRNAs, including 174 transcription 


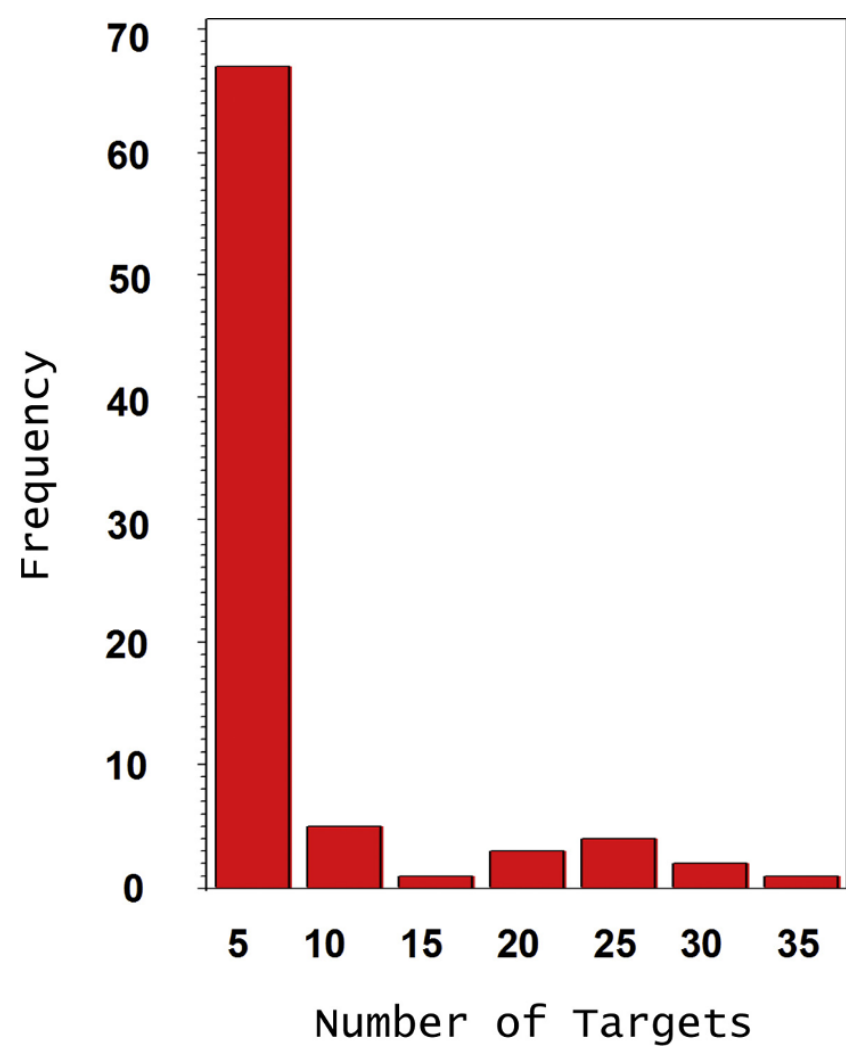

Figure 4

The frequency distribution of predicted targets for the various miRNAs. The frequency distribution of the number of target genes predicted per miRNA using the inhouse developed plant target prediction program.

factors. Among 78 miRNA, 29 miRNAs have one predicted target. TAG_1080847 has the most number with 37 predicted targets, including 12 AP2 domain transcription factors (Figure 4). Figure 5 shows the complementary alignment between the miRNA and the predicted target gene sequences. We selected some miRNAs and conducted 5' RACE experiments to validate the cleavage site and miRNA predicted targets. Figure 6 shows examples of the cleavage site for miRNA TAG_5113378 and its target gene Glyma18g05330 encoding a putative ARF transcription factor.

\section{Soybean Genome Browser and Solexa transcriptomics data integration}

A comparison of the small RNA and cDNA expression data within the soybean genome browser enables rapid correlations between specific miRNA and their predicted target genes. Figure 7 shows the miRNA and small RNA library sequence distribution across the entire genome, one entire chromosome at a time. It also allows visualization of the miRNA overlapping against the

$\begin{array}{lcc}\text { TAG_3208888 } & \text { Glyma13g05300.1 } & 5 \\ \text { miRNA } 24 & \text { UACGUCGAAGAGCAAACCAGAAAG } & 1 \\ & ::::::::::::::::: & \\ \text { Target 2727 } & \text { AUCCGGCUUCUUGUUUGGCCUCUC } & 27 \\ & & \\ \text { TAG_5248950 } & \text { Glyma14g39170.1 } & 5 \\ \text { miRNA 22 } & \text { ACUAUAACCGUGCCGAGUUAGU } & 1 \\ & :::::::::::::::: & \end{array}$

Target 456 UGGUGUUGGCACGGUUGGCUCA

\section{Figure 5}

Sequence alignment between miRNA and its predicted target genes. The alignment between the miRNA and predicted target gene sequences where ":" indicates a perfectly complementary base and "." indicates a $\mathrm{G}: \mathrm{U}$ wobble.

\section{TAG_5113378 \\ Glyma18g05330}

\section{Figure 6}

miRNA target gene cleavage site validated by

5"RACE. miRNA TAG_5II3378 cleavage site on its target gene Glyma I8g05330 as identified by 5' RACE is highlighted by red arrow. In the alignment ":" indicates a perfectly complementary base and “.” indicates a G:U wobble.

soybean release version gene model predictions. The Solexa transcriptomics data add another level of information about the expression of the soybean genes in three of the same tissues used for the miRNA analysis. We omitted the comparison between the seed-derived miRNA and the green pod transcriptomic data since these two conditions are likely not comparable.

\section{Discussion}

The soybean genome browser developed utilizing the architecture provided by the UCSC Genome Browser allows for incorporation of the small RNA data, along with soybean transcriptome sequencing data. Overlaying these two datasets along side the soybean gene model predictions facilitates a complete view of the small RNA library distribution along entire chromosomes in one view. It also allows biologists to compare the miRNA expression levels to that of the predicted target genes to get valuable insight into the regulation patterns. 


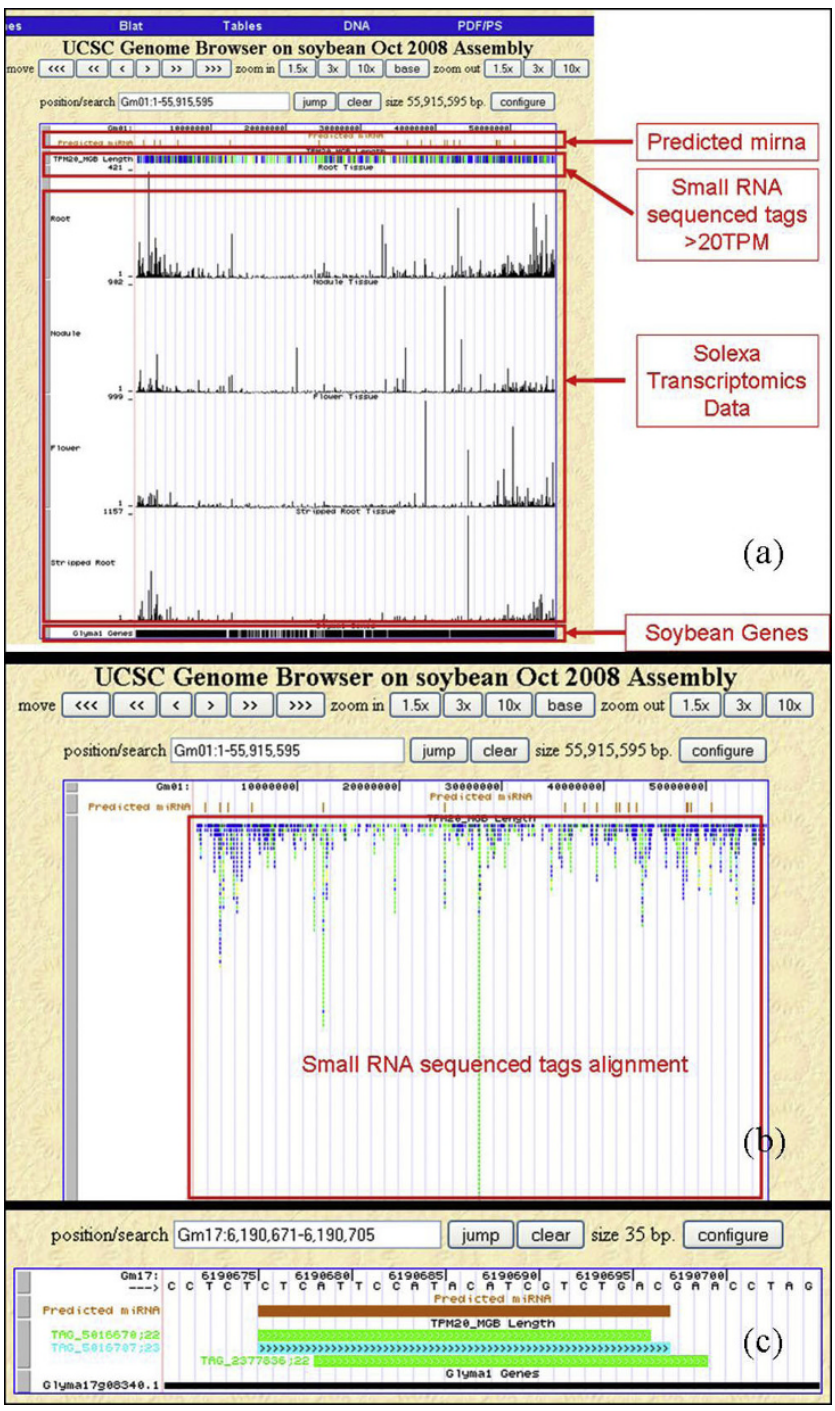

\section{Figure 7}

Soybean Genome Browser. The soybean genome browser displaying (a) the predicted miRNA sequences, small RNA library sequences $>20$ TPM and Solexa transcriptomics data from four tissues mapped onto chromosome I; (b) full display of small RNA library sequences >20 TPM; and (c) TAG_5016707 predicted miRNA and the surrounding other mapped sequences.

In order to investigate the target gene regulation patterns, we calculated the Spearman correlation coefficients [29] between the miRNA expression levels and the respective target gene expression levels. The miRNAs will degrade their target genes, so miRNA expression should show a negative correlation with the respective target gene. However, some miRNAs could also be co-transcribed with their host genes and targets, especially for miRNAs that are located in intronic regions and self-regulate their host genes. If this is the case, then the expression of some
miRNAs will show a positive correlation with their targets [30]. In our results, we observed some strong negative-correlations between miRNAs and their targets (Figure 8a). At the same time, we also observed some positive correlations between the two expression patterns. These results are similar to that reported by Wang for human cancer cells [31]. The density distribution of the correlation coefficients provides a full view of the relationship between miRNAs and their targets (Figure $8 \mathrm{~b}$ ). It shows that there are more cases of negative correlation between miRNAs and their targets than positive correlation. Dugas et al. [32] documented at least one Arabidopsis miRNA, miR172, which reduced the accumulation of target protein without significantly effecting target mRNA levels, suggesting that this miRNA may play a role in inhibiting productive translation without affecting mRNA levels. Very little is known about transcriptional and post-transcriptional regulation
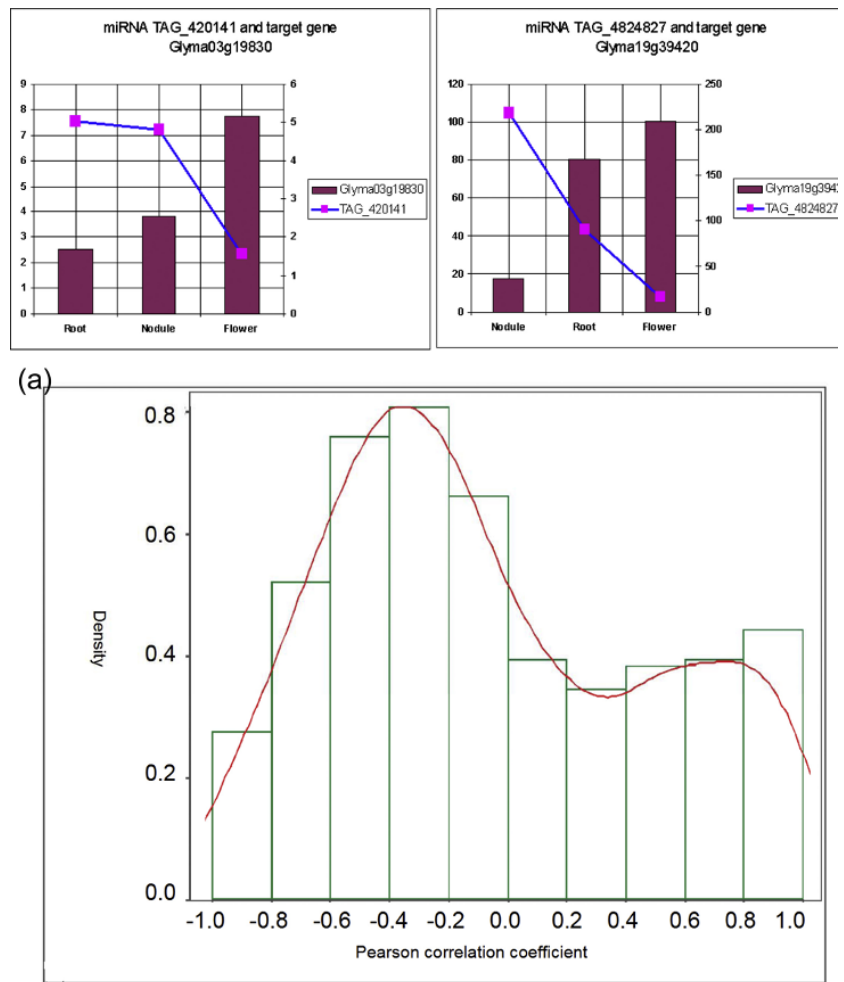

(b)

\section{Figure 8}

miRNA and target gene pair regulation. (a) miRNA and its target gene pair show a strong negative Spearman correlation coefficient between their expression patterns. This strong negative correlation suggests the down regulation of the target genes in response to miRNA expression. (b) The density distribution of correlation coefficient between the miRNA and their target genes expression patterns. 
of miRNAs and, obviously, there is still much to learn. Few studies have sought an understanding of the miRNA complex regulation process in plants and we intend to further expand our observations to studying this relationship by utilizing the unique datasets available to us in soybean.

\section{Conclusion}

Sequencing of four small RNA libraries in soybean which generated over one million sequencing reads per library and its subsequent bioinformatics analyses to identify the authentic known and novel miRNA added 87 new miRNA to the list of known soybean miRNAs. This study encompasses many more soybean tissues than those examined by earlier studies and also provides a unique opportunity to study the relationship between the miRNA expression levels and their regulation of the corresponding target genes utilizing the Solexa cDNA sequencing data derived from the same tissues at the same time. The visualization of the small RNA libraries data alongside the transcriptomics data in the genome browser can help biologists to better understand the dynamics of gene regulation. The many hypothesis generated from this relationship can help advance our understanding of miRNA target gene regulation in the future. As more miRNAs are discovered and their target genes identified, finding biological roles for these interactions enables deeper understanding of gene regulation and of the roles of both the miRNA and its target genes in plant development.

\section{Competing interests}

The authors declare that they have no competing interests.

\section{Authors' contributions}

TJ performed the bioinformatics analysis, developed the in house scripts for the miRNA and the target gene identification, analyzed the Solexa transcriptomics data, deposited the soybean datasets in the genome browser and drafted the initial manuscript. ZY and ML identified target genes, performed hairpin structure prediction and contributed in the discussion of this manuscript. DJ and SP were involved in RNA isolation and library construction. PJG contributed to the experimental design and small RNA libraries. DJS was involved in plant sample production and sequencing of small RNA libraries. AF and GM were involved in Solexa cDNA sequencing. BCM contributed to the experimental design and target prediction. DX provided guidance on the computational analyses. GS conceived the study and obtained funding for the experimental studies. All authors read and approved the final manuscript.

\section{Additional material}

\section{Additional file 1}

Predicted miRNA list identified in soybean. The list of all 129 predicted miRNA and the miRNA family assigned to those matching already known miRNA.

Click here for file

[http://www.biomedcentral.com/content/supplementary/14712105-11-S1-S14-S1.doc]

\section{Acknowledgements}

TJ and DX were supported by United Soybean Board. ZY, ML and GS were supported by a grant from the National Science Foundation, Plant Genome Research Program, \#DBI-0421620). Work on legume small RNAs in the labs of PJG, DJS and BCM was supported by USDA award 2006-03567. We would also like to thank Robin Kramer for setting up and maintaining the genome browser locally, where the data of this study were deposited.

This article has been published as part of BMC Bioinformatics Volume II Supplement I, 2010: Selected articles from the Eighth Asia-Pacific Bioinformatics Conference (APBC 2010). The full contents of the supplement are available online at http://www.biomedcentral.com//47I2105/II?issue=SI.

\section{References}

I. Brodersen $P$ and Voinnet $O$ : The diversity of RNA silencing pathways in plants. Trends Genet 2006, 22:268-280.

2. Lippman Z and Martienssen R: The role of RNA interference in heterochromatic silencing. Nature 2004, 43 I:364-370.

3. Zhang BH, Pan XP, Cannon CH, Cobb GP and Anderson TA: Identification and characterization of new plant microRNAs using EST analysis. Cell Res 2005, | 5:336-360.

4. Carrington JC and Ambros V: Role of microRNAs in plant and animal development. Science 2003, 30I:336-338.

5. Glazov EA, Cottee PA, Barris WC, Moore RJ, Dalrymple BP and Tizard ML: A microRNA catalog of the developing chicken embryo identified by a deep sequencing approach. Genome Res 2008, 18:957-64.

6. He L and Hannon G]: MicroRNAs: Small RNAs with a big role in gene regulation. Nat Rev Genet 2004, 5:522-531.

7. Chapman EJ and Carrington JC: Specialization and evolution of endogenous small RNA pathways. Nat Rev Genet 2007, 8:884-896.

8. Meyers BC, Axtell MJ, Bartel B, Bartel DP, Baulcombe D, Bowman JL, Cao X, Carrington JC, Chen X and Green PJ, et al: Criteria for annotation of plant MicroRNAs. Plant Cell 2008, 20:3।86-3190.

9. Hannon GJ: RNA interference. Nature 2002, 4I 8:244-25I.

10. Matzke M, Matzke AJM and Kooter JM: RNA: guiding gene silencing. Science 2001, 293:1080-1083.

II. Zhu JK: Reconstituting plant miRNA biogenesis. PNAS 2008, I05:985I-9852.

12. Subramanian S, Fu Y, Sunkar R, Barbazuk WB, Zhu JK and Yu O: Novel and nodulation-regulated microRNAs in soybean roots. BMC Genomics 2008, 9:160-174.

13. Boualem A, Laporte P, Jovanovic M, Laffont C, Plet J, Combier JP, Niebel A, Crespi M and Frugier F: MicroRNAI 66 controls root and nodule development in Medicago truncatula. Plant J 2008, 54:876-887.

14. Combier JP, Frugier F, de Billy F, Boualem A, El-Yahyaoui F, Moreau S, Vernie T, Ott T, Gamas P and Crespi M, et al: MtHAP2-I is a key transcriptional regulator of symbiotic nodule development regulated by microRNA169 in Medicago truncatula. Genes Dev 2006, 20:3084-3088.

15. Phytozome. http://www.phytozome.net/soybean.

16. Lullien V, Barker DG, de Lajudie P and Huguet T: Plant gene expression in effective and ineffective root nodules of alfalfa (Medicago sativa). Plant Mol Biol 1987 9:469-478.

17. Nobuta K, Lu C, Shrivastava R, Pillay M, De Paoli E, Accerbi M, Arteaga-Vazquez M, Sidorenko L, Jeong $\mathrm{DH}$ and $Y e n ~ Y$, et al: 
Distinct size distribution of endogenous siRNAs in maize: Evidence from deep sequencing in the mop I-I mutant. PNAS 2008, 105:|4958-14963.

18. Zhang Z, Schwartz S, Wagner L and Miller W: A greedy algorithm for aligning DNA sequences. I Comput Biol 2000, 7:203-|4.

19. Lowe TM and Eddy SR: tRNAscan-SE: A program for improved detection of transfer RNA genes in genomic sequence. Nucl Acids Res 1997, 25:955-964.

20. Chan PP and Lowe TM: GtRNAdb: A database of transfer RNA genes detected in genomic sequence. Nucl Acids Res 2008, 37 Database: D93-D97.

21. Lagesen K, Hallin PF, Rodland E, Staerfeldt HH, Rognes $T$ and Ussery DW: RNammer: consistent annotation of rRNA genes in genomic sequences. Nucleic Acids Res 2007, 35:3100-8.

22. Meyers BC, Tej SS, Vu TH, Haudenschild CD, Agrawal V, Edberg SB, Ghazal $\mathrm{H}$ and Decola S: The use of MPSS for whole-genome transcriptional analysis in Arabidopsis. Genome Res 2004, I 4: I64I-I653.

23. Markham NR and Zuker M: DINAMelt web server for nucleic acid melting prediction. Nucleic Acids Res 2005, 33:W577-W58I.

24. Griffiths-Jones S, Saini HK, van Dongen S and Enright AJ: miRBase: tools for microRNA genomics. Nucleic Acids Res 2008, 36 Database: DI54-DI58.

25. Higgins D, Thompson J, Gibson T, Thompson JD, Higgins DG and Gibson TJ: CLUSTAL W: improving the sensitivity of progressive multiple sequence alignment through sequence weighting, position-specific gap penalties and weight matrix choice. Nucleic Acids Research 1994, 22:4673-4680.

26. Allen E, Xie Z, Gustafson AM and Carrington JC: microRNAdirected phasing during transacting siRNA biogenesis in plants. Cell 2005, I21:207-221.

27. Schwab R, Palatnik JF, Riester M, Schommer C, Schmid M and Weigel D: Specific effects of microRNAs on the plant transcriptome. Developmental cell 2005, 8:517-27.

28. Maq: Mapping and Assembly with Qualities. http://maq. sourceforge.net/index.shtml.

29. Hogg RV and Craig AT: Introduction to Mathematical Statistics.New York: Macmillan; 51995, 338-400.

30. Baskerville $S$ and Bartel DP: Microarray profiling of microRNAs reveals frequent coexpression with neighboring miRNAs and host genes. RNA 2005, I I:24I-247.

31. Wang YP and $\mathrm{Li} K \mathrm{~KB}$ : Correlation of expression profiles between microRNAs and mRNA targets using NCI-60 data. BMC Genomics 2009, 10:218-231.

32. Dugas DV and Bartel B: MicroRNA regulation of gene expression in plants. Current Opinion in Plant Biology 2004, 7: 512-520.
Publish with BioMed Central and every scientist can read your work free of charge

"BioMed Central will be the most significant development for disseminating the results of biomedical research in our lifetime. "

Sir Paul Nurse, Cancer Research UK

Your research papers will be:

- available free of charge to the entire biomedical community

- peer reviewed and published immediately upon acceptance

- cited in PubMed and archived on PubMed Central

- yours - you keep the copyright
BioMedcentral 